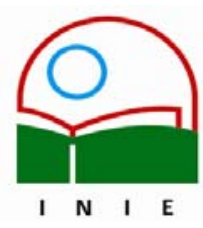

Universidad de Costa Rica

Facultad de Educación

Instituto de Investigación en Educación

ACTUALIDADES INVESTIGATIVAS EN EDUCACION

\title{
LA EVALUACIÓN EDUCATIVA: CONCEPTO, PERÍODOS Y MODELOS
}

\section{Ana Isabel Mora Vargas ${ }^{1}$}

Resumen: El propósito es ofrecer al lector un recorrido por los modelos que han fundamentado la evaluación educativa, lo que permite reflexionar acerca de la evaluación como una estrategia útil y necesaria para el mejoramiento de la calidad de la educación. Se inicia el artículo con el concepto de evaluación, funciones y normas. En la exposición se continúa ubicando la evaluación en cuatro períodos o generaciones. Estos temas constituyen las bases para desarrollar el tema de los enfoques evaluativos que han tenido influencia en el ámbito educativo. Este artículo se deriva del marco conceptual que sustenta un proyecto de investigación acerca de la evaluación del plan de estudios en la Universidad de Costa Rica, inscrito en el Instituto de Investigación para la Educación (INIE), antiguo IIMEC. Para complementar la información bibliográfica se incluye aspectos de la experiencia acumulada de la autora, producto de los procesos de investigación participativa que se han realizado con las unidades académicas. En otras publicaciones se ofrecerán al lector los lineamientos metodológicos para evaluar el plan de estudios que surgen de la investigación.

Palabras claves: EVALUACIÓN/ PERÍODOS DE EVALUACIÓN/ ENFOQUES DE EVALUACIÓN Y MODELOS DE EVALUACIÓN/

Abstract: The purpose is to offer the reader an overview of the models that have served as a foundation of the educative evaluation, which allows to reflect upon the evaluation as a useful and necessary strategy for the improvement of the quality of the education. The article starts with the concept of evaluation, its functions and standards. Evaluation is categorized in four periods or generations. These topics deal with the evaluation approaches that have had influence on the education environment. This article derives from the conceptual framework that supports an investigation project about the evaluation of the curriculum in the University of Costa Rica, registered in the Institute of Research in Education (INIE), previously known as IIMEC. In order to complement the bibliographical information, it includes the author's accumulative experience on collective research. In future publications, the methodological guidelines will be offered to the reader in order to evaluate the curriculum that arises from the investigation.

Key words: EVALUATION/ PERIODS OF EVALUATION/ EVALUATION APPROACHES AND MODELS OF EVALUATION/

El propósito de este artículo es ofrecen diferentes posiciones teóricas acerca del concepto de evaluación educativa, las funciones y las normas que pueden marcar la diferencia entre el tipo de evaluación que se elija. También se realiza un recorrido por los períodos o las llamadas generaciones de la evaluación y por los modelos de la evaluación educativa. El sustento bibliográfico que ofrece el artículo es complementado con la

\footnotetext{
1 Magister Scientiae en Educación con énfasis en Investigación Educativa. Licenciada en Ciencias de la Educación con énfasis en Currículo. Bachiller en Enseñanza Especial con énfasis en Trastornos de la Comunicación. Profesora de la Escuela de Formación Docente de la Facultad de Educación de la Universidad de Costa Rica. Investigadora del Instituto de Investigación en Educación (INIE). Miembro de la Comisión de Investigación y Trabajos Finales de Graduación. Correo electrónico: aimora@cariari.ucr.ac.cr
}

Artículo recibido: 24 de agosto, 2004

Aprobado: 13 de diciembre, 2004 
experiencia obtenida por la autora, en investigaciones inscritas en el Instituto de Investigaciones Educativas (INIE), antiguo IIMEC, realizadas tanto en el nivel de educación primaria como en el nivel de educación superior. En todas las investigaciones se realizan procesos participativos donde se involucra a docentes, estudiantes, personal administrativo y técnico, especialmente.

El artículo se organiza en tres apartados: a) concepto de evaluación, b) Periodos de la evaluación y c) modelos de la evaluación educativa.

\section{A.- Concepto de evaluación}

La evaluación se puede entender de diversas maneras, dependiendo de las necesidades, propósitos u objetivos de la institución educativa, tales como: el control y la medición, el enjuiciamiento de la validez del objetivo, la rendición de cuentas, por citar algunos propósitos. Desde esta perspectiva se puede determinar en qué situaciones educativas es pertinente realizar una valoración, una medición o la combinación de ambas concepciones.

Algunas definiciones presentan una orientación meramente cuantitativa de control y medición del producto, se pueden concebir como "una fase de control que tiene como objeto no sólo la revisión de lo realizado sino también el análisis sobre las causas y razones para determinados resultados, ...y la elaboración de un nuevo plan en la medida que proporciona antecedentes para el diagnóstico". (Duque, 1993, p. 167). A la vez, la evaluación está orientada por una teoría institucional (leyes, reglamentos, decretos y circulares) y por la cultura evaluativa existente, entendida como la forma que se han realizado los procesos evaluativos. Ésta "se construye a través del conjunto de valores internalizados por docentes, alumnos, directores, supervisores padres y representantes de entes empleadores, acerca de la forma de concebir y practicar la evaluación en un determinado proceso educativo." (Duque, 1992, p. 170).

El Joint Committee on Standards for Educational Evaluation señala que "la evaluación es el enjuiciamiento sistemático de la validez o mérito de un objeto" (Stufflebeam y Shinkfield, 1995, p. 19). De tal manera, que en un estudio es importante tanto lo bueno como lo malo de la situación evaluada, de lo contrario no se trata de una evaluación. En consecuencia, Stufflebeam y Shinkfield (1995, p. 20), consideran que la evaluación es un proceso complejo pero inevitable. Es una fuerza positiva cuando "sirve al progreso y se utiliza para identificar los puntos débiles y fuertes, y para tender hacia una mejora". Las 
bases de valoración que deben considerarse al evaluar algo son: expectativas de los usuarios, mérito del servicio y hasta qué punto éste es necesario. Además, se deben considerar otros aspectos de la evaluación: así como la viabilidad y la equidad y si requiere ser comparada, centrándose en un producto o servicio. También se debe tener claridad en las principales utilizaciones tales como: el perfeccionamiento, la recapitulación y la ejemplificación y hasta qué punto los evaluadores requieren cualificaciones especiales.

Otra posición señala a la evaluación como una herramienta para la rendición de cuentas. El concepto no es solo rendir cuentas de los aciertos y desaciertos de un plan o programa de estudios o del desempeño profesional, sino también recibir retroalimentación para el mejoramiento académico y personal tanto del personal docente como de la población estudiantil y, por ende, de la institución educativa. La evaluación educativa, se puede considerar como un instrumento para sensibilizar el quehacer académico y facilitar la innovación (González y Ayarza, 1996).

En consecuencia, “...todo proceso que se asuma como evaluación institucional tiene como requisito y condición indispensable la participación de la comunidad educativa...de allí que la evaluación tenga como característica fundamental la auto-evaluación" (González y Ayarza, 1996). La evaluación realizada sólo por agentes externos a la vida institucional tiende a fracasar, dado que no contempla el desarrollo de un proceso participativo con las personas que componen la comunidad educativa, limitando su participación a ofrecer datos posiblemente mediante instrumentos de preguntas cerradas.

Finalmente se cita la definición de López (1995), la cual sustenta a la evaluación curricular en el manejo de información cualitativa y cuantitativa para juzgar el grado de logros y deficiencias del plan curricular, y tomar decisiones relativas a ajustes, reformulación o cambios. Igualmente permite verificar la productividad, la eficacia y la pertinencia del currículo.

La búsqueda de alternativas a la solución de problemas es el reto fundamental de la evaluación como proceso para el mejoramiento de la calidad de la educación. Para ello es necesario crear un clima organizacional donde se facilite y propicie la práctica evaluativa. En los procesos participativos que ha realizado la autora en sus proyectos de investigación, el clima organizacional de la evaluación se inicia con la indagación de la forma en que las personas interesadas entienden el proceso evaluativo y como se han llevado a cabo otras evaluaciones anteriores; es decir, que ha sido lo positivo de esos procesos y qué se debe mejorar. Este paso se considera necesario, dado que la experiencia ha demostrado que las personas no están dispuestas a emprender procesos participativos sino encuentran 
motivación y justificación para cambiar las prácticas tradicionales de la evaluación. También se indaga acerca de sus necesidades, expectativas y del compromiso que se asume ante el desarrollo de la evaluación. Luego se plantea un anteproyecto, se analiza con las personas interesadas y se realizan los ajustes pertinentes. En un proyecto participativo es necesario definir los niveles de participación de las personas involucradas y trabajar coordinadamente, es importante que se sientan parte del proceso y de la toma de decisiones.

El tipo de evaluación que la institución elija se relaciona con el propósito de la misma; seguidamente se hace referencia a las funciones y a las normas de la evaluación.

\section{Funciones de la evaluación}

Cualquier tipo de evaluación que se realice en el ámbito educativo, debe cumplir con funciones como las que se citan a continuación (Posner, 1998; Hernández, 1998; Díaz Barriga, 1999):

Función de diagnóstico: La evaluación de un plan o programa de estudios debe caracterizar el planeamiento, ejecución y administración del proyecto educativo, debe constituirse en síntesis de sus principales aciertos y desaciertos. De tal manera, que le sirva a las autoridades académicas de orientación o de guía que permita derivar acciones tendientes al mejoramiento de la calidad de la educación.

Función instructiva: El proceso de evaluación en sí mismo, debe producir una síntesis de los indicadores de la puesta en práctica del currículum. Por lo tanto, las personas que participan en el proceso, se forman, aprenden estrategias de evaluación e incorporan una nueva experiencia de aprendizaje laboral.

Función educativa: A partir de los resultados de la evaluación donde el personal docente conoce con precisión cómo es percibido su trabajo por sus iguales, por el estudiantado y por las autoridades académicas de la institución, puede trazarse una estrategia para erradicar las insuficiencias que le han señalado en su desempeño profesional. Por lo tanto, existe una importante relación entre los resultados de la evaluación del plan o programa de estudios y las motivaciones y actitudes del personal docente hacia el trabajo.

Función autoformadora: Esta función se cumple principalmente cuando la evaluación ofrece lineamientos para que la persona responsable de la docencia oriente su quehacer académico, sus características personales y para mejorar sus resultados. Poco a poco la persona se torna capaz de autoevaluar crítica y permanentemente su desempeño, no teme a sus errores, sino que aprende de ellos y es más consciente de su papel como responsable de diseñar y ejecutar el currículum. Desarrolla habilidad en cuanto a lo que sabe y lo que no 
sabe y necesita conocer; de manera que desarrolla la necesidad de autoformación tanto en el plano profesional como en el desarrollo personal. El carácter formador de la evaluación, por si solo, justifica su necesidad.

La función autoformadora es la persigue la autora en los procesos evaluativos, de la experiencia obtenida se puede concluir que es un proceso difícil dado que se presentan obstáculos tales como: la resistencia al cambio, el poco interés en asumir compromisos de participación y el miedo a enfrentar nuevos retos. La evaluación es lenta y debe ser un proceso de análisis y reflexión constante, hasta lograr un cambio de actitud ante las situaciones problemáticas que se están evaluando y mejorando. Cuando el análisis y reflexión del desempeño docente, por ejemplo; se convierte en una práctica diaria, se empieza a notar el mejoramiento en la calidad de la enseñanza.

Además de las funciones de la evaluación es necesario señalar las normas básicas de la evaluación.

\section{Normas para la evaluación}

El Joint Committee on Standards for Educational Evaluation y la Evaluation Research Society han formulado dos conjuntos de normas para la evaluación (Stufflebeam y Shinkfield, 1995). El trabajo de estas organizaciones ha sido guiado por dos premisas básicas; la primera señala que la evaluación es una actividad humana esencial e inevitable. La segunda, se fundamenta en que una evaluación solvente proporciona una comprensión más amplia y una mejora de la educación.

En general, las normas del Joint Committee "aconsejan que los evaluadores y las personas involucradas en este proceso cooperen entre sí para que las evaluaciones puedan cumplir cuatro condiciones principales" (Stufflebeam y Shinkfield (1995, pp. 26-27):

- Ser útil al facilitar informaciones acerca de virtudes y defectos así como soluciones para mejorar.

- Ser factible al emplear procedimientos evaluativos que se puedan utilizar sin mucho problema.

- Ser ética al basarse en compromisos explícitos que aseguren la necesaria de cooperación, la protección de los derechos de las partes implicadas y la honradez de los resultados

- Ser exacta al describir el objeto en su evolución y contexto, al revelar virtudes y defectos, al estar libre de influencias y al proporcionar conclusiones. 
Estas normas pueden aplicarse en cualquier etapa del proceso evaluativo, ya sea al decidir si hay que realizar la evaluación, al planearla y llevarla a cabo, al presentar los resultados y aplicar las conclusiones. En los procesos de evaluación participativa que ha realizado la autora, se parte analizando las funciones de factibilidad y ética. La función de utilidad está muy ligada a la de exactitud, lo que depende, en gran medida, del nivel de compromiso y responsabilidad con que se desarrollo la evaluación.

En la evolución del concepto e evaluación se distinguen varios períodos que responden a generaciones, a continuación se abordan elementos que nos ubican los diferentes períodos.

\section{B.- Períodos de la evaluación}

De acuerdo con la revisión bibliográfica, los períodos de la evaluación se ubican en cuatro generaciones: medición, descriptiva, juicio y constructivista. La generación de la medición incluye el período pre-tyleriano, la generación descriptiva el período tyleriano, la generación de juicio contempla los períodos de la inocencia y el realismo y, en la cuarta generación se ubican los períodos del profesionalismo y autoevaluación.

Período pre-tyleriano. Este es el período más antiguo, como dato curioso se cree que este tipo de evaluación data aproximadamente del año 2000 a.C. Autores como Guba y Lincoln (1989), consideran que este período se inicia a finales del siglo pasado y sigue vigente. Tiene relevancia a finales del siglo pasado, en los Estados Unidos, con el inicio del movimiento para la acreditación de instituciones y programas educativos y, en los primeros años de este siglo, con la aparición de los tests estandarizados como instrumentos de medición y evaluación (Rama 1989). Se ha caracterizado por "centrar la atención de la calidad de la educación en el rendimiento de los estudiantes, medición que se realiza mediante tests o pruebas"; a este período se ha denominado "primera generación: de la medición". (Dobles, 1996, p. 80)

Período tyleriano. En los primeros años de la década de los treinta, Ralph Tyler presenta una renovada visión del currículo y la evaluación. Plantea el modelo de planificación curricular tecnológico, en el cual pone énfasis en la selección y organización del contenido, así como en las estrategias para transmitir la información y evaluar el logro de los objetivos. Para Tyler, la evaluación determina en que medida han sido alcanzados los objetivos y para ello, sugiere realizar comparaciones entre los resultados y los objetivos propuestos en un programa de estudios (Tyler, 1974 y Rama, 1989). De acuerdo con la clasificación de Guba y Lincoln (1989), se le llama "segunda generación: descriptiva". 
Surge después de la Primera Guerra Mundial; en este período se considera insuficiente una evaluación centrada en el rendimiento del estudiante por lo que optan por "una evaluación que se caracteriza por una descripción de patrones que evidencien fortalezas y debilidades en relación con objetivos establecidos." (Dobles, 1996, p. 81)

Período de la "inocencia". A finales de la década de los cuarenta y durante los primeros años de la década de los cincuenta, en los Estados Unidos se hace evidente una expansión de las ofertas educacionales y, por consiguiente se incrementa la práctica de la evaluación del personal docente y de la evaluación educacional (Rama, 1989). En este periodo prevalecen los principios propuestos por Tyler para la evaluación educativa, centrados en la medición de los resultados.

Período del realismo. En los años 60, en los Estados Unidos, la evaluación se comienza a profesionalizar y, a raíz de ese fenómeno, en educación se plantea la necesidad de elaborar nuevos proyectos para el desarrollo del currículo; especialmente, en ciencias y matemática. Crombach recomienda que se reconceptualice la evaluación "como un proceso consistente en recoger y formalizar información que pueda ayudar a quienes elaboran los currículos" (Rama, 1989, p. 38). En la clasificación de Guba y Lincoln (1989), este periodo aparece después de 1957 y se le denomina "tercera generación: de juicio". Este se caracteriza por "los esfuerzos para enriquecer los juicios, en donde el evaluador asume el papel de juez, aunque mantiene el de técnico, así como también las funciones descriptivas..." (Dobles, 1996, p. 81).

Período del profesionalismo. A partir de la década del 70 , la evaluación empieza a tomar auge como una profesión que relaciona la evaluación con la investigación y el control (Rama, 1989, González y Ayarza, 1997).

Período de autoevaluación. En las últimas décadas, con la proliferación de instituciones de educación superior, en Latinoamérica, se ha visto la necesidad de competir por calidad académica. Se parte de la premisa que todos los esfuerzos educativos, incluyendo la evaluación deben buscar el crecimiento cognitivo y el desarrollo personal de todos los participantes en el procesos de enseñanza y aprendizaje (Hernández, 1998). Esto conlleva a proceso de autoevaluación con miras a la acreditación de carreras profesionales. También se considera la metaevaluación como medio para asegurar y comprobar la calidad de las evaluaciones. En este sentido, la evaluación se concibe como orientadora y formativa del proceso; de tal manera que, se convierte en uno de los mejores procedimientos del control de la calidad de la educación (Hernández, 1999). De acuerdo con la nomenclatura de Guba y Lincoln (1989), este período constituye la "cuarta generación: constructivista". 
"En las generaciones anteriores, los parámetros han sido construidos a priori...". En la llamada cuarta generación "los límites y parámetros los construyen las personas que participan en la evaluación, como elemento importante dentro del mismo proceso de evaluación", se considera que en el campo de la acreditación no encuentra evidencias que permitan afirmar que se ha entrado a la cuarta generación (Dobles, 1996, p. 81).

Cada período ha estado acompañado de reformas curriculares que responden a las necesidades del contexto sociocultural y laboral del momento.

Reformas curriculares. En las reformas más recientes se destacan requisitos básicos para enfrentarlas (Imbernón, 1991; Villarini, 1996, Posner, 1996; Yus, 1998, Hernández, 1998):

- Una reforma curricular sólo funciona cuando las personas involucradas: docentes, estudiantes, entre otras, participan activamente en el proceso. De esta manera, conocen, interiorizan y asumen una actitud racional ante el compromiso que tiene la institución educativa con la sociedad en cuanto al desarrollo científico, cultural, político y axiológico.

- En las reformas curriculares es necesario considerar la necesidad de desarrollar estrategias para la capacitación docente. También es necesario buscar mecanismos para que esta capacitación trascienda a procesos de autoformación de docentes en servicio. Con el desarrollo de esas actividades, los profesionales de la docencia, puede generar cambios de actitud hacia la enseñanza de su disciplina, lo que sin duda, redunda en la formación integral de los nuevos profesionales. Algunos aspectos a considerar:

- Asume, con sentido de investigador, permanente el enfoque y el diseño curricular que lo caracteriza como docente. Esto permite una relación de alcance y secuencia entre la asignatura que imparte y las demás asignaturas del plan de estudios.

- Interioriza el papel del docente actual, el cual se concibe como mediador en los procesos de construcción del conocimiento y que para ello es necesarios asumir el aprendizaje continuo y permanente como una forma de vida.

- Asume, con visión prospectiva, los alcances de la globalización del conocimiento y los cambios culturales del mundo contemporáneo. Esto le exige al docente diseñar nuevas estrategias dentro de nuevos paradigmas educativos y culturales que respondan al reto de un desarrollo humano integral, sustentable y auto sostenido, que permita la supervivencia del equilibrio ecológico, mental y natural.

- Para que la evaluación tenga valor formativo para todos los participantes en las acciones evaluadoras, es imprescindible el conocimiento, análisis y debate conjunto de las evidencias que afloren durante el proceso de evaluación. 
Desde este punto de vista, la evaluación es considerada como un instrumento estratégico para el mejoramiento de la gestión y la calidad de la educación así como un instrumento para dar garantía al público sobre la seriedad de los programas de estudio que se están poniendo en práctica. A continuación se presentan los modelos de evaluación que han tenido influencia en Latinoamérica.

\section{C.- Modelos de evaluación}

En este apartado se consignan los principios teóricos de las posiciones que han adoptado los sistemas de educación latinoamericanos en los últimos años. Los modelos han tenido sus épocas de auge y han evolucionado de acuerdo con los períodos y las reformas educativas. En la actualidad, gran cantidad de estudios consultados, señalan a la evaluación participativa y la evaluación fundamentada en competencias, como estrategias para el cambio.

Para Stufflebeam y Shinkfield (1995), la evaluación tiene el propósito de enjuiciar o perfeccionar el valor o el mérito de un objeto. En sus estudios acerca de diferentes modelos de evaluación, los agrupa en diferentes categorías: la pseudoevaluación, la cuasievaluación, la evaluación verdadera y la evaluación holística.

1.- La pseudoevaluación o evaluaciones políticamente orientadas en las que se intenta conducir a determinadas conclusiones mediante una evaluación. Distinguen dos tipos:

- Las investigaciones encubiertas donde la intención del cliente es obtener, mantener o incrementar una esfera de influencia, poder o dinero.

- Los estudios basados en las relaciones públicas cuya intención es ayudar al cliente a crear una imagen positiva del objeto evaluado.

2.- La cuasievaluación o evaluación en la que se trata de responder a ciertas cuestiones de interés, en lugar de determinar el valor de algo. Existen diferentes tipos de cuasievaluaciones tales como: la comprobación de programas, los sistemas de información administrativa, los estudios de responsabilidad, los estudios basados en objetivos y los estudios basados en la experimentación. Destacan los últimos dos tipos:

Los estudios basados en objetivos. Se parte de objetivos fijados por las personas interesadas o por el conjunto de participantes en el proceso. El propósito más común es 
determinar si los objetivos se han cumplido. Este tipo de estudio es el que más frecuentemente se utiliza con el nombre de evaluación. Dentro de las críticas que se le hacen a este método cabe resaltar que la información llega demasiado tarde para ser utilizada en el perfeccionamiento de los servicios, y que esta información es, a menudo, de muy poco alcance como para constituir una base sólida a la hora de enjuiciar el valor de un servicio (Stufflebeam y Shinkfield, 1995, p. 71). Por lo tanto, en algunas situaciones es necesaria la evaluación dirigida al control y medición y, en otros a la de rendición de cuentas.

Los autores consideran a Ralph Tyler como el pionero de este tipo de estudios. Desarrolló el primer método sistemático de evaluación educacional como parte de su trabajo en los años treinta y principios de los cuarenta. Considera que la evaluación "debe determinar la congruencia entre trabajo y objetivos" Para este autor, el procedimiento para evaluar un programa involucra (Stufflebeam y Shinkfield, 1995, p. 92):

- Establecer metas u objetivos. Tyler no los diferenciaba; sin embargo, se puede inferir de sus escritos que las metas eran ideales y los objetivos eran submetas.

- Ordenar los objetivos en amplias clasificaciones.

- Definir los objetivos en términos de comportamiento.

- Establecer situaciones y condiciones según las cuales se puede demostrar la consecución de los objetivos.

- Explicar los propósitos de la estrategia al personal más importante.

- Escoger o desarrollar las técnicas evaluativas apropiadas.

- Recopilar los datos de trabajo.

- Comparar los datos con los objetivos de comportamiento.

Este método ha sido de gran impacto en la educación, ya que la evaluación educacional se había centrado casi exclusivamente en el estudiante, con el aporte de Tyler, se atienden otros aspectos del programa. Al respecto, proporciona medios prácticos para la retroalimentación mediante la reformulación o redefinición de objetivos. La estrategia tyleriana ofrece la oportunidad que "el evaluador con iniciativa pudiera examinar los datos más relevantes del proceso mediante el cual se desarrollaba el programa", con el propósito de resaltar la importancia y la utilidad de la evaluación tanto para juzgar el proceso de un programa como sus resultados finales. Esto nunca fue promulgado por Tyler ni puesto en práctica por sus seguidores contemporáneos, sino que lo utilizaron otros posteriormente, como Scriven en la evaluación formativa y Stufflebeam en la evaluación del proceso (Stufflebeam y Shinkfield, 1995, pp. 94-97). 
También se han señalado algunas limitaciones a este método, tales como:

- La evaluación se ha utilizado casi exclusivamente para valorar el logro final; es decir, como proceso terminal que no ofrece la oportunidad para perfeccionar el programa durante el proceso evaluativo. Esto, a pesar de que teóricamente resalta el principio de retroalimentación y de su utilización en la mejora de la educación.

- En el modelo no se especifica como se debe realizar la selección y clarificación de los objetivos.

- Aunque el modelo tyleriano pone énfasis especial en los objetivos relacionados con el rendimiento y señala, teóricamente, que debe atender tanto los procedimientos educativos como a los estudiantes; éste se centra especialmente en el rendimiento de los estudiantes.

Los estudios basados en la experimentación. Este modelo se ha clasificado dentro de los tipos de cuasievaluación, dado que incluye metodologías que permiten "el juicio de valores" (Stufflebeam y Shinkfield, 1995, p. 71). Se destacan la exposición de problemas, la hipótesis y el estudio de las cuestiones. Los problemas, generalmente son planteados por investigadores o dirigentes y no por participantes del proceso. Su principal fin es determinar o demostrar vínculos causales entre ciertas variables. Entre los pioneros en el uso de la experimentación para evaluar programas están Suchman, 1967 y Cronbach y Snow, 1969 (Stufflebeam y Shinkfield, 1995). La principal ventaja de este tipo de evaluación consiste en el aporte de “... métodos sólidos para la determinación de las relaciones causales, relativamente inequívocas entre el programa y los resultados". No obstante, algunos problemas relacionados con el método se señalan con frecuencia, así por ejemplo, se indica que proporciona información mucho más restringida de la que es necesaria para evaluar programas en el ámbito educativo y que la "...información final que no resulta útil como guía para el proceso de desarrollo" (Stufflebeam y Shinkfield, 1995, p. 72). Este tipo de evaluación todavía tiene gran influencia en la concepción de evaluación de los educadores, romper estos esquemas es una de las principales causas de resistencia al cambio, con que se ha encontrado la autora en las investigaciones realizadas.

3.- La evaluación verdadera cuyo fin es enjuiciar o perfeccionar el valor o mérito de un objeto. Se distinguen, varios tipos, los más relevantes se exponen a continuación: 
- Los estudios para la toma de decisiones. Se parte de la necesidad de tomar decisiones y su intención básica es proporcionar conocimientos y bases valorativas para tomar y justificar decisiones. Entre los métodos adecuados para llevar a cabo este tipo de estudios están las inspecciones, valoración de las necesidades, estudios de casos, series de recomendaciones, observaciones estructuradas y planificaciones cuasiexperimentales y experimentales. Entre los evaluadores que consideran que la evaluación debe contribuir a la toma y justificación de decisiones están Cronbach y Stufflebeam, (Stufflebeam y Shinkfield, 1995). En la evaluación para la toma de decisiones la principal característica es "utilizar la evaluación continúa y sistemática para planificar y llevar a cabo los servicios que satisfacen las necesidades del cliente" También se puede señalar que la principal limitación es que la colaboración entre el evaluador y el que toma las decisiones puede conducir a una distorsión de los resultados. Para contrarrestar estas posibles desviaciones se puede recurrir a la metaevaluación externa, es decir, a la evaluación de la evaluación (Stufflebeam y Shinkfield, 1995, p. 73).

- Los estudios centrados en el cliente. Se centran principalmente en ayudar a quienes realizan un servicio cotidiano tendiente a valorar y perfeccionar sus contribuciones. Los problemas planteados proceden de la comunidad, de grupos locales y de expertos ajenos al caso. En este sentido, se trata de una investigación activa que orienta a quienes realiza programas a dirigir sus propias evaluaciones. La principal desventaja que se le señala es la "ausencia de credibilidad externa y la posibilidad de ser manipulado por ciertos elementos locales que, en efecto, ejercen un gran control sobre la evaluación" (Stufflebeam y Shinkfield, 1995, p. 74).

Los métodos más usados son el estudio de casos, informes contrapuestos y la evaluación respondente. El pionero en este tipo de estudios es Stake (1967); con la propuesta de evaluación respondente, el autor presenta una alternativa frente a diferentes métodos evaluativos, tales como: el modelo pre-test y post-test (evaluación preordenada), el modelo acreditativo (autoestudio y visita de expertos), la investigación aplicada al modelo didáctico (Cronbach), la evaluación orientada hacia el consumidor (Scriven), la evaluación orientada hacia la decisión (Stufflebeam), la metaevaluación (Scriven), la evaluación sin metas (Scriven) y la evaluación contrapuesta (Owens y Wolf).

El propósito de la evaluación respondente es proporcionar un servicio dirigido a personas específicas; en este sentido, el evaluador debe conocer el lenguaje e intereses de su audiencia. Desde esta perspectiva, la evaluación se concibe como "un valor observado, 
comparado con alguna norma", teniendo como principales propósitos la descripción y el juicio de un programa. La tarea básica del evaluador consiste en presentar un informe detallado de "lo que ha observado acerca del programa y de la satisfacción que siente un grupo de personas adecuadamente seleccionado para el programa" (Stufflebeam y Shinkfield, 1995, p. 252).

- Los estudios políticos. Sirven para identificar y valorar los méritos de diversas políticas que compiten en una sociedad o segmento social. Se considera a Joseph Rice (1900), el pionero de este enfoque. Los métodos usados incluyen la Técnica Delphi, la planificación experimental y cuasiexperimental, los guiones, los pronósticos y los procedimientos judiciales. La principal ventaja de este estilo de evaluación es que “... resultan esenciales como guía de las instituciones y de la sociedad. Su principal inconveniente es que, una vez más, acaban corrompidos o subvertidos por el ambiente político en el que se desarrollan" (Stufflebeam y Shinkfield, 1995, p. 75).

Han colaborado en este enfoque, entre otros, Owens y Wolf (1972), que presentan el método contrapuesto de evaluación, investigan los pro y los contra de un programa con el fin de clarificar sus principales problemas. La intención final de este método es proporcionar evidencias sólidas a quienes toman las decisiones. Este tipo de evaluación representa "un gran paso hacia el final de una etapa que empezó con el método orientado hacia los objetivos de Tyler" (Stufflebeam y Shinkfield, 1995, p. 293). Este modelo ha tomado diversas formas; por ejemplo, el modelo judicial de R. Wolf, en el cual se trata de establecer un acuerdo en el contexto de un desacuerdo. Puede funcionar conjuntamente con otros modelos propuestos tales como el modelo de Scriven, Stufflebeam y Provus.

El modelo de la contraposición se desarrolla como respuesta a lo inadecuado de la evaluación basada en tests psicológicos para proporcionar información para la toma de decisiones. En el contexto legal, los procedimientos de contraposición juzgan, mediante una audiencia administrativa, los méritos de un caso en el que están implicadas dos partes opuestas. Estos procedimientos pueden ser aplicados para la toma de decisiones en educación.

Dentro de los usos principales del modelo se pueden señalar las siguientes (Stufflebeam y Shinkfield, 1995):

- Explorar los valores de un currículo nuevo o existente.

- Seleccionar nuevos libros de texto.

- Estimar la congruencia entre una innovación educativa y el sistema existente. 
- Revelar las interpretaciones hechas por distintos representantes sobre los mismos datos.

- Informar el procedimiento evaluativo, principalmente a las autoridades educativas y a las personas involucradas como docentes, estudiantes y las personas encargadas de la administración.

- Resolver las disputas acerca de los contratos de trabajo.

- Tomar decisiones para poner en práctica estrategias de mejoramiento.

Los estudios basados en el consumidor. El propósito de este enfoque es “... juzgar los valores relativos de los bienes y servicios alternativos $y$, como consecuencia, ayudar a los contribuyentes y clientes a tener criterios para elegir y adquirir esos bienes y servicios". Los métodos incluyen listas de control, valoración de las necesidades, evaluación de los objetivos, planificación experimental y cuasiexperimental, análisis del "modus operandi” y el análisis de los costes. Dentro de las ventajas resalta el hecho de que se trata de una “...valoración sólida e independiente concebida para proteger a los consumidores...”. Su principal desventaja es que puede llegar a independizarse de quienes lo practican que quizá no pueda ayudarles a la hora de servir mejor a los consumidores, además requiere de un experto en el tipo de recursos que atiende al consumidor (Stufflebeam y Shinkfield, 1995, pp. 75-76).

El primero en aplicarlo a la educación es Scriven (1967), quien concibe la evaluación como "la determinación sistemática y objetiva del valor o el mérito de algún objeto", recalca que la meta es siempre la misma "juzgar el valor", en forma objetiva. Considera que el proceso evaluativo se debe basar en un análisis comparativo de los costes y efectos de los objetos con los competidores críticos, especialmente las alternativas más económicas. En este sentido, el autor señala que la evaluación debe atender tanto a los costes y efectos del objeto como a la satisfacción de la necesidades de los consumidores. Destaca dos funciones principales: la evaluación formativa y la sumativa. La evaluación formativa suministra información pertinente para la planificación y su posterior producción de algún objeto. También contribuye a que el personal de la institución perfeccione cualquier actividad que este realizando. Indica que en la elaboración del curriculum, permite la solución de algunos problemas tales como: la validez del contenido, el nivel del vocabulario, la utilidad de los medios, la durabilidad de los materiales y la eficiencia, entre otros aspectos. La evaluación sumativa suministra información acerca del valor del objeto después de haber sido desarrollado y puesto en el mercado (Stufflebeam y Shinkfield, 1995, pp. 241-247). 


\section{4.- El método holístico de la evaluación.}

Estas propuestas son consideradas por Stufflebeam y Shinkfield (1995), como verdaderas evaluaciones, a pesar de que reconocen la ausencia de credibilidad externa. En este apartado se han incluido los modelos que conciben, en sus principios teóricos, a la evaluación como un proceso de valoración del estado total del objeto de estudio. Se citan los más relevantes:

La evaluación orientada hacia el perfeccionamiento. Este modelo responde a las demandas del trabajo de Stufflebeam como evaluador educativo en los Estados Unidos de Norte América (1960-70). El autor considera que la evaluación tyleriana no era adecuada ya que los resultados de la misma se conocen al final del proceso cuando es demasiado tarde para resolver sus problemas; además señala que la definición de evaluación propuesta por Tyler se limita a determinar si los objetivos han sido alcanzados. En consecuencia, propone redefinir el concepto de evaluación como el "proceso mediante el cual se proporciona información útil para la toma de decisiones" y plantea el modelo CIPP (Contexto-entradaproceso-producto) Stufflebeam y Shinkfield (1995, pp. 176-208).

Al respecto se mencionan algunos aspectos relevantes en las cuatro fases del modelo de evaluaciónde Stufflebeam y Shinkfield (1995):

- Evaluación del contexto. En este tipo de evaluación, los objetivos principales son la valoración del estado global del objeto, ya sea la institución, el programa, la población o la persona en relación con las deficiencias, virtudes, problemas y características del marco global en que se desenvuelve. También, se puede examinar si las metas y prioridades existentes están en consonancia con las necesidades que debe satisfacer el objeto. Los resultados de la evaluación deben proporcionar los lineamientos para ajustar o realizar los cambios necesarios en las metas y prioridades del proyecto evaluado. La metodología puede incluir gran variedad de valoraciones del objeto y diferentes tipos de análisis. Generalmente, el punto de partida consiste en entrevistar a las personas que solicitan el estudio para conocer su opinión en relación con los defectos, virtudes y problemas. Esto permite plantear hipótesis acerca de los cambios necesarios. Otros aspectos metodológicos incluyen construcción de instrumentos de estudio tales como: encuestas, análisis de informes existentes, test diagnóstico, técnica para llegar a consenso y, talleres de análisis y reflexión, entre otros. 
- Evaluación de entrada (input). El objetivo central es ayudar a prescribir un programa para efectuar los cambios necesarios La evaluación ayuda a identificar y valorar los métodos aplicables, especialmente los que ya se están ejecutando así como los métodos que se selecciona para su aplicación o continuación. La metodología que se emplea requiere de la revisión del estado de la práctica con respecto a la satisfacción de necesidades. Inicialmente, se puede estudiar de la literatura, contactar programas ejemplares y consultar a expertos. Posteriormente, un grupo especial debe ordenar y analizar esta información para proponer soluciones, definir criterios para una renovación y estimar recursos y barreras que inciden en las posibilidades de cambio.

- Evaluación del proceso. Este tipo de evaluación consiste en la comprobación permanente de la realización de un plan o proyecto. Se plantea como objetivos, proporcionar a las personas encargadas de la administración y al personal de la institución, información continua acerca de la ejecución del programa y de la guía para modificar o explicar el plan. Desde esta perspectiva, la persona responsable del proceso evaluativo se convierte en el eje central de la evaluación y a una o más personas se les debe asignar las tareas de realizar revisiones sobre la marcha y de buscar la documentación e información continua, que pueda ayudar a realizar el programa tal y como se ha planeado. Para este fin se recurre a técnicas como: la observación de actividades del programa, reuniones informativas regulares, informes, entre otras estrategias.

- Evaluación del producto. El propósito es valorar, interpretar y juzgar los logros o la satisfacción de necesidades del programa; así como los efectos deseados y no deseados. El trabajo se puede valorar en relación con algunas normas previamente escogidas, se pueden emplear variedad de técnicas que incluyen: audiciones o entrevistas en grupo para generar hipótesis acerca de los resultados; investigaciones clínicas para confirmar o rechazar hipótesis; estudios de casos concretos para obtener una visión profunda de los efectos; interrogar por teléfono o correo a una muestra de participantes; pedir a los participantes que presenten ejemplos concretos, entre otros. En el diseño de la evaluación de procesos es importante destacar los siguientes criterios:

- En el análisis de la tarea es necesario que evaluado y evaluador cooperen.

- El plan para la obtención de información básicamente debe ser desarrollado por la persona encargada de la evaluación. 
- En el informe de los resultados débe señalarse la participación de los involucrados en el proceso evaluativo.

- También es necesaria una amplia colaboración en el plan para la administración del estudio. Así como también reviste gran importancia evaluar las evaluaciones; es decir, realizar la metaevaluación que abarca, entre otros, los criterios, procesos y técnicas empleadas para evaluar evaluaciones.

- Para que una evaluación sea solvente debe abordar las cuestiones adecuadas, ser exactas en sus definiciones, ser comprensibles y ser justas para las personas que están siendo evaluados.

- La evaluación iluminativa. En 1972 se reúnen en conferencia un grupo de 14 investigadores con experiencia en prácticas evaluativas, para buscar alternativas al modelo evaluativo de Tyler, entre los más destacados se pueden citar a Robert Starke por Estados Unidos, David Hamilton, Marcolm Parlett y Barry MacDonald por Gran Bretaña. La principal crítica al modelo de Tyler, basado en objetivos es que valora sin explicar. Por lo tanto, la conferencia recomienda un replanteamiento total de las bases lógicas y las técnicas de los programas evaluativos existentes que condujo al desarrollo de la evaluación iluminativa. Para los autores, los métodos evaluativos convencionales han seguido las tradiciones experimentales o psicométricas cuyo alcance es limitado e inadecuado para tratar los problemas complejos que enfrenta quien tiene a cargo el proceso de evaluación. Consideran que los intentos de valoración de los productos educativos deben dejar paso a un "estudio intensivo del programa como totalidad: su base lógica, su evolución, sus operaciones, sus logros y sus dificultades", la "metodología iluminativa contempla el programa como un todo", dándole énfasis a la descripción cualitativa del fenómeno y menos a la descripción cuantitativa (Stufflebeam y Shinkfield, 1995, p. 313).

El modelo denominado evaluación iluminativa es propuesto por Parlett y Hamilton (1977); el objetivo principal es la descripción y la interpretación, más que la valoración y la predicción. Se intenta plantear y clarificar una serie de cuestiones que ayuden a las partes interesadas a identificar los aspectos y procedimientos del programa que permitan lograr los resultados deseados. Estos autores consideran que la nueva propuesta evaluativa requiere no sólo del cambio de metodologías sino también de nuevas suposiciones y conceptos para comprenderla desde dos aspectos: el sistema de enseñanza y el medio de aprendizaje. Este tipo de evaluación consiste en una estrategia de investigación general, que se base en un problema de investigación; por lo tanto, el evaluador se debe familiarizar con la realidad 
cotidiana del objeto de investigación. En una investigación curricular se pone énfasis en la observación en las aulas así como en las entrevistas con docentes, estudiantes y participantes. No obstante, se utilizan otras técnicas para la recopilación de datos en otras, tales como: los cuestionarios, los tests y el análisis de contenido de fuentes documentales. Se distinguen tres etapas de una evaluación iluminativa Parlett y Hamilton (Stufflebeam y Shinkfield, 1995): la fase de observación, en la cual se investigan todas las variables que afectan el resultado del programa. La fase de investigación, en la que se seleccionan los aspectos más importantes del programa en su contexto y, la fase de explicación donde se exponen los principios generales subyacentes a la organización y operación del programa.

El propósito principal de los estudios evaluativos es contribuir a la toma de decisiones. E informe del evaluador está dirigido a tres grupos responsables de tomarlas: participantes en el programa, patrocinadores o administradores del programa, los grupos externos interesados y, los investigadores y comités escolares. El formato del informe, en la evaluación iluminativa no es relevante, siempre y cuando sea legible, breve e interesante. Cada grupo toma decisiones distintas, es por ello que no es suficiente emitir un juicio dicotómico, funciona o no funciona el proyecto o programa evaluado. La persona responsable de la evaluación se debe comunicar con la audiencia de una manera más natural y efectiva que en la evaluación tradicional.

Aunque también critica la posición evaluativa tradicional, Stenhouse expresa algunas reservas acerca del método iluminativo: existe preocupación por el "mérito " o "valor" de un currículo o una práctica educativa pero sus criterios no son claros, y su preocupación por las audiencias y la presentación de los resultados parece enmascarar este problema. Opina que un elemento importante en la evaluación de un currículo es la crítica filosófica que revela su significado más que calcula su valor. Parlett y Hamilton reconocen que la amplia utilización de técnicas abiertas, de progresiva concentración y datos cualitativos sigue permitiendo gran parcialidad. Sin embargo, sugieren que ésta se puede contrarrestar al poner en duda las interpretaciones preliminares, al considerar inevitable el efecto de quien evalúa el desarrollo y la evolución del programa (Stufflebeam y Shinkfield, 1995, pp. 327328).

- La evaluación constructivista. No se encuentran modelos de evaluación sistematizados como los citados anteriormente; sin embargo el constructivismo como tendencia evaluativa ha tomado auge en los últimos años, especialmente en la evaluación de proyectos sociales. En el ámbito educativo y curricular, la evaluación constructivista debe 
partir de un plan o programa de estudios fundamentado en procesos. En este sentido, cabe señalar que existe la posibilidad que se cuente con algunas limitaciones para la puesta en práctica de ese tipo de evaluación. Es casi imposible suponer que la totalidad de personal docente desarrollen la práctica educativa desde la perspectiva constructivista. Por otra parte, en la práctica educativa surge gran variedad de problemáticas y es posibles que no todos los problemas se puedan resolver desde esta perspectiva. Por lo tanto, la evaluación de este tjpo de proceso educativo es más complejo, el evaluador en general, no dispone de instrumentos y estrategias adecuadas para abordar la evaluación. Tomando en cuenta que los problemas educativos nunca tienen una única causa ni radican exclusivamente en un sólo aspecto del plan o programa de estudios o del proyecto educativo. En este caso es difícil señalar un método o técnica evaluativa; es recomendable la perspectiva sistémica, ésta permite contextualizar a la institución educativa dentro de un sistema, en interacción con otros sistemas; de manera tal, que cualquier cambio en un elemento exige cambios en el sistema.

Este tipo de evaluación constructivista se ha puesto en práctica en proyectos sociales, se parte de una concepción de evaluación como "el juicio emitido -de acuerdo a ciertos criterios preestablecidos- por una persona o un equipo sobre las actividades y resultados de un proyecto" (Román, 1999). Se propone realizar un análisis sistemático, lo más objetivo posible acerca de las distintas etapas y resultados alcanzados por los proyectos, considerando aspectos tales como: la pertinencia y logro de objetivos, la eficiencia, el impacto y sustentabilidad de las acciones. Se fundamenta en una metodología sistémica que contempla la relación entre el contexto, los insumos, los procesos y los productos o resultados del proyecto para describirlo y explicarlo (Stufflebeam y Shinkfield, 1995 y Román, 1999). En consecuencia, se asume "el proyecto como un sistema de acción, cuyos elementos interactúan entre sí y con su entorno, tanto para generar como para aceptar cambios" (Román, 1999). Se considera que este enfoque permite visualizar la relación y consistencia entre los distintos componentes de la estrategia asumida por el proyecto y el efecto que estos tienen en los distintos ámbitos o niveles en los que interviene. El enfoque presenta un cambio de paradigma epistemológico sistémico-constructivista que "... traslada el foco de la atención desde el ser de las cosas observadas al observador como sistema" (Román, 1999, p. 7). La función principal del investigador social es observar, describir y explicar observaciones; por lo tanto, la técnica más relevante es la observación, considerándose el investigador social como un observador externo u observador de segundo orden. Desde esta posición el observador puede determinar el qué y cómo observan y 
operan los sujetos observados. Desde esta perspectiva ha toma auge la denominada autoevaluación, que consiste en un proceso de análisis y reflexión introspectivo y prospectivo acerca del propio quehacer educativo, necesario para mejorar el servicio que brinda una institución educativa.

En la autoevaluación deben participar los miembros de la comunidad educativa, tales como: estudiantes, docentes, autoridades académicas, personal administrativo y de apoyo técnico, entre otras participaciones de interés para el proyecto. Debe concebirse como una evaluación integral que analiza profundamente el plan o programa de estudios en sus distintas etapas: planteamiento, ejecución y administración, además permite delinear un plan de trabajo para realizar las modificaciones que sean necesarias.

La educación superior costarricense tanto pública como privada esta entrando en la cultura de autoevaluación para su posterior acreditación. La acreditación es un proceso de mejoramiento continuo inspirada en los principios de eficacia, eficiencia, oportunidad, pertinencia. Para llegar a la acreditación la institución educativa debe someterse a diferentes etapas de evaluación, en primer lugar debe pasar por la autoevaluación, la evaluación externa, nacional e internacional y finalmente la acreditación. Entendido éste como un proceso de credibilidad y legitimidad de parte de la sociedad representada por organizaciones, empresas, instituciones, etc.

En la educación primaria y secundaria todavía no se ha entrado en proceso de acreditación, pero si en inquietudes por realizar procesos de autoevaluación. Como investigadora y docente de la Universidad de Costa Rica, la autora ha impartido charlas sobre este tema en diferentes instituciones de primaria, también se ha asesorado a estudiantes que realizan trabajos finales de graduación que muestran interés por los procesos de autoevaluación para la educación secundaria.

- La evaluación fundamentada en competencias. Este enfoque ha recobrado importancia, dado que la competitividad, la productividad, la calidad de los procesos y productos son los retos para el cambio que marcan el crecimiento económico y productivo; razón por la cual, la educación debe buscar otros criterios para lograr el desarrollo de las capacidades como las habilidades y destrezas innovadoras que le permitan al profesional enfrentar los retos que impone la nueva realidad. Este enfoque ha evolucionado en sus planteamientos teóricos, dando paso a la perspectiva constructivista; por lo tanto, desde el ámbito de la planificación curricular pone atención no solo a la formación académica sino también al desarrollo humanos, especialmente en momentos en que se cuestiona la visión 
de ser humano debido a que diversos factores convergen en la deshumanización, tales como: la globalización, los avances científicos y tecnológicos, el consumismo, la pobreza económica, social y cultural, la xenofobia y la deforestación entre otros aspectos (Stufflebeam y Shinkfield,1995 y Román, 1999).

Una evaluación por competencia requiere de un planteamiento de diseño de plan de estudios elaborado por competencias En este contexto competencia se puede entender como el conocimiento, las actitudes y las destrezas necesarias para desempeñar una ocupación dada. La manera como los sistemas educativos están abordando la competencia laboral difiere en cada país, y depende de las necesidades del mercado laboral y de la evolución que ha tenido el proyecto educativo para incorporar una formación basada en competencias. En Costa Rica no se conocen aplicaciones de este enfoque en la educación superior. No obstante, algunos docentes entrevistados, mencionan que los planes de estudios deben dar énfasis al desarrollo de habilidades para la competencia profesional. Al respecto se tienen datos de planes de estudio fundamentados en competencias en Colombia; en el nivel de educación secundaria técnica como el Covao y el CIPET han empleado modelos basados en normas de competencia.

Las competencias se han clasificado de diferentes maneras respondiendo a las necesidades de formación de cada institución, cada autor presenta la clasificación de acuerdo con el diseño de plan de estudios. Los siguientes tres tipos de competencias se han organizado a partir de la reflexión teórica basada en los estudios de: Mertens, 1996 y 1998, Bogoya, 2000; Jiménez, 2000, Corpi, 2001, Morales 2001. La clasificación presenta tres tipos de competencias generales que constituyen los ejes o áreas propuestas para un diseño de plan de estudios. Éstas a su vez se pueden descomponer en subcompetencias que se distribuyen en los ciclos lectivos, de acuerdo con los niveles de secuencia y profundidad del contenido.

Competencias profesionales. En este tipo de competencias se pueden distinguir dos niveles, las cuales varían según las características particulares del plan de estudios:

- Competencias laborales genéricas. Esto es, saberes y destrezas generales que son aplicables a una gama de ocupaciones relativamente amplia.

- Competencias ocupacionales específicas, o saberes y destrezas que demanda cada ocupación en particular.

Competencias de formación humana, se refieren a los valores y actitudes apropiados para desenvolverse en ambientes laborales, el énfasis de esta competencia radica en el desarrollo de la personalidad del profesional. Las competencias de formación humana apuntan hacia el 
logro de una formación ética sólida, realista y práctica a la vez y a promover el desarrollo de personas capaces de respetar y hacer respetar los valores institucionales y los principios éticos, tanto en sus vidas privadas, como en el desempeño profesional. Con este tipo de competencias se pretende el desarrollo pleno del ser humano. Mediante las actividades y contenidos curriculares y extracurriculares tanto los docente como los estudiantes se preocupan por encontrarle sentido a su vida individual y social, el entendimiento de sí mismo y de quienes lo rodean; se autoevalúan y se vuelven conductores de su propio proyecto de vida. En este sentido, el plan de estudios debe prever espacios de interacción y de encuentro interpersonal que rescaten y promuevan valores, e incrementar el diálogo como estrategia para la solución de conflictos; asimismo generar espacios de participación comunitaria y de amistad. De manera que, el futuro profesional se desarrolle y se prepare para contribuir al cambio y a la transformación social. Las competencias de apoyo se consideran un nivel de esta competencia.

- Competencias de apoyo, se relacionan con los aportes de diferentes áreas académicas que contribuyen a la formación y desarrollo profesional.

Las Competencias académicas se refieren directamente a la formación académica y pretenden desarrollar en los estudiantes un alto nivel académico, una adecuada interacción entre la teoría y la práctica, un alto grado de conceptualización, reflexión y análisis crítico e interpretación de los hechos; con base en esa formación, el profesional sea capaz de proponer soluciones e innovaciones en su entorno socio cultural.

Dentro de este tipo de competencias se pueden distinguir las competencias de informática. Con esto se pretende que la informática no sea considerada únicamente un elemento que facilite la realización de una labor, sino que se convierta en una herramienta para la selección y el manejo de la información, con el propósito de alcanzar la eficacia y eficiencia necesarias en el desempeño profesional. La informática también es un medio de evolución profesional. Otra competencia es la investigación. En las tendencias curriculares actuales la investigación constituye una actividad básica en el aprendizaje, genera una práctica de indagación formativa que debe manifestarse de manera concreta, en el trabajo desarrollado entre los docentes y los estudiantes. La actividad investigativa tiene como punto de partida la producción de conocimientos en función de las necesidades individuales y profesionales tomando en cuenta los principios éticos y de exigencia profesional.

También se distinguen subcompetencias en diferentes niveles de profundidad del conocimiento. Por ejemplo, las subcompetencias de nivel 1 son conocimientos o destrezas que debe tener el profesional para alcanzar la Competencia General; las subcompetencias 
de nivel 2 son las habilidades y conocimientos que se deben tener para lograr las de nivel 1, y así sucesivamente, hasta establecer el nivel más específico de tareas y funciones básicas, requeridos para alcanzar la Competencia General propuesta. A su vez las competencias generales se pueden descomponer en subcompetencias que se distribuyen en los ciclos lectivos, con secuencia y profundidad. En este sentido, la organización de los cursos debe plantearse por competencias y no por temáticas como en los cursos tradicionales.

Por lo tanto, el diseño metodológico para evaluar el plan de estudios fundamentado en competencias debe contemplar esta modalidad desde la definición del perfil académicoprofesional En el perfil fundamentado en competencias se centra en el área profesional o laboral, tratando que el área humanística y de apoyo logren equilibrar la formación del profesional. Antes de elaborar los programas de los cursos es necesario verificar el perfil profesional, previamente elaborado mediante consultas a los académicos de la carrera, a la población estudiantil, a los representantes de entes empleadores entre otros; es decir, se contextualiza el programa. La estructura de un plan de estudios por competencias necesita un modelo de evaluación diferente al tradicional, dado que las competencias propuestas tienen dos opciones se alcanzan o no se alcanzan las competencias en forma integral, no tendría sentido afirmar, por ejemplo que un nivel de subcompetencia se alcanza y que la competencia general se alcanza parcialmente o no se alcanza.

Como consideración final se puede llamar la atención a que en el proceso de cambio se pueden distinguir diferentes posiciones. Por una parte, se presenta el cambio como necesario para que el sistema esté en posición de contribuir efectivamente al desarrollo nacional. Se afirma que los sistemas educativos pueden contribuir al desarrollo y consolidación de los sistemas democráticos y, a la construcción de una sociedad alternativa (Rama, 1989). Por otra parte, se considera el cambio como un requisito indispensable para asegurar la supervivencia institucional. Aquellos que impulsan esta posición advierten que la situación actual para las instituciones educativas es tal, que no se pueden resistir al cambio, de lo contrario no están en condiciones de competir ni de legitimarse frente a la sociedad (Soria, 1997).

En las instituciones de educación superior se hace evidente la preocupación por el mejoramiento de la calidad de la educación, especialmente con la gran proliferación de universidades en los últimos años. Actualmente se han acreditado carreras tanto de la Universidad de Costa Rica y las otras universidades públicas como de las universidades privadas. En la Universidad de Costa de Costa Rica, el Centro de Evaluación Académica (CEA), ha estado asesorando los procesos de autoevaluación de las carreras interesadas en 
la acreditación. Para ello ofrece a la comunidad universitaria el documento denominado "El proceso de Autoevaluación para la Acreditación. Una guía orientadora", (CEA, 1999). Esta guía presenta seis componentes del proceso de autoevaluación a saber: factor (plan de estudios, docencia, personal académico, población estudiantil, organización y administración, recursos físicos, materiales y financieros), criterios de evaluación de la calidad de la carrera (coherencia, eficacia, eficiencia, equidad, idoneidad, impacto, integridad, pertinencia, relevancia, responsabilidad, transparencia e universalidad), indicadores que permitan verificar la calidad de los factores, pauta que indican el nivel en que se requiere trabajar el indicador, fuentes (documentos, graduado, empleadores, estudiantes, docentes, director o decano, personal administrativo y otros) y acciones y preguntas generadoras sugeridas, estas las propone el CEA con el propósito de acceder al cumplimiento del indicador. Este último apartado tiene como propósito que las unidades académicas puedan relacionar las acciones y preguntas con los criterios, indicadores y pautas determinados por el Sistema Nacional de Acreditación de la Educación superior (SINAES). Por su parte el SINAES, presenta a las instituciones interesadas en la acreditación, el "Manual de Acreditación" (SINAES, 2000). Los aspectos básicos de este proceso de autoevaluación integral se relacionan con la institución educativa y con la carrera o programas tales como: personal académico, curriculum, estudiantes, administración, infraestructura y equipamiento y, el impacto y pertinencia del curriculum de la carrera.

Es importante mencionar que en la Universidad de Costa Rica cada día son más las carreras que se abocan a los procesos de autoevaluación con miras a acreditación. Hasta agosto del 2004 se han acreditado las siguientes carreras: Licenciatura en Medicina y Cirugía (Sede Central), Licenciatura en Trabajo Social (Sede Central), Licenciatura en Biología. Licenciatura en Farmacia y Licenciatura en Psicología (CONARE, SINAES, 2004). La autora ha tenido la oportunidad de participar como lectora en proceso de autoevaluación de carreras con miras a la acreditación y, con base en la experiencia se puede señalar que los procesos evaluativos que emprenden las carreras los llevan a un análisis y reflexión profunda de su práctica educativa y, de las acciones que deben emprender en aras del mejoramiento de la calidad de la educación. En los procesos realizados por las carreras se evidencian procesos participativos que involucran docentes, estudiantes, autoridades académicas y entes empleadores, especialmente. Se señalan las fortalezas y las estrategias para la solución de las debilidades. Sin duda, que estas acciones y otras que surgen como producto de la autoevaluación, marcan la diferencia en la calidad de la educación. 


\section{Referencias}

Alfaro, Gilberto. (1996). Evaluación cualitativa: técnicas y estrategias. San José, Costa Rica: UNA.

Arríen, Juan. (1997). Calidad y acreditación exigencias a la universidad. La Educación Superior en el Siglo XXI- Visión de América Latina y el Caribe- Tomo I. CRESALC-UNESCO.

Ayarza, Hernán. (1994). Calidad y acreditación universitaria. Modelos de acreditación. Canadá, Organización Universitaria Interamericana, Instituto de Gestión y Liderazgo Universitario: Revista IGLU, No.6, Abril.

Badilla, Leda (Compiladora). (1996). Tendencias Actuales en la Medición y Evaluación Educativa: Memoria del Foro Taller Internacional. San José, Costa Rica: Oficina de Publicaciones de la Universidad de Costa Rica.

Bogoya, Daniel. (2000). Evaluación de competencias. En Revista Javeriana. Abril.

Bok, Derek. (1990). Universities and the Future of America. Durham, N.C.: Duke University Press.

Castillo, Jonathan. (2000). Estrategias docentes para el aprendizaje significativo. Recuperado de www.monografías.com

CONARE. Sistema Nacional de Acreditación de la Educación Superior (SINAES). (2000). Manual de Acreditación convocatoria del año 2000. San José, C.R.: CONARE, OPES.

(2000). Modificaciones al Manual de Acreditación Edición Año 2000. San José, C.R.: CONARE, OPES.

Carreras acreditadas por el SINAES. Recuperado de
http://www.conare.ac.cr/sinaes/carreras.htm (1990). Leyes, convenios y decretos de la educación superior universitaria estatal en Costa Rica. San José, C.R.: CONARE, OPES.

Copri, J. Elizabeth; Ortiz, Sergio. La Educación Basada en competencias. México: Publicaciones de la UIA-Noroeste.

CSUCA. (1997). Sistema centroamericano de evaluación y acreditación de la educación superior, SICEVAES. La Educación Superior en el Siglo XXI- Visión de América Latina y el Caribe- Tomo I. Caracas, CRESALC-UNESCO, 1997.

De Alba, Alicia. (1996). Análisis de Discurso Educativo Curricular: Una Propuesta de Evaluación Curricular. México: UNAM. 
Dell Ordine, José Luis. (2000). La evaluación educativa. Club Caminantes- Pedagogía. Buenos Aires, Argentina: BANFIELD-PCIA.

Díaz Barriga, Angel. (1998). Evaluación Académica: Organismos Internacionales y Política Educativa. México: UNAM.

Díaz Barriga, Angel. (1992). Docentes, planes y programas de estudio e institución educativa. Perfiles Educativos, \#57-58, Centro de Investigaciones y servicios educativos de la UNAM, México.

Dobles, María Cecilia. (Directora Doctorado Educación UCR). (1996). Acreditación en educación superior: orígenes, prácticas actuales, perspectivas. Comunicación personal.

Duque, R. (1993). La evaluación en la ES Venezolana. Planiuc. Números 17-18, Aniversario X.

Farbara, E. (1996). Situaciones de los Sistema Educativos en América Latina. Santa Fe de Bogotá: Editorial Guadalupe. Convenio Andrés Bello (CAB).

Garita, Luis. (1995). Ceremonia de inauguración. Seminario: Evaluación de la calidad y gestión de cambio. Evaluación de la calidad y gestión del cambio. Pallán y Van der Donckt (editores). México: ANUIES.

Gento Palacios, Samuel. (1998). Marco referencial para la evaluación de un proyecto educativo. Revista: Educación XXI, \# 1. Madrid: EUNED.

González, Luis E.; Ayarza, Hernán. (1997). Calidad, evaluación institucional y acreditación en la educación superior en la región Latinoamericana y del Caribe. Documento central. La educación superior en el siglo XXI. Visión de América Latina y el Caribe. Documentos de la Conferencia Regional Políticas y Estrategias para la Transformación de la Educación Superior en América Latina y el Caribe, La habana, Cuba, 1996. Caracas: CRESALC-UNESCO.

Hernández, Pedro. (1998). Diseñar y Enseñar. Madrid: NARCEA.

Imbernón, F.; (coord.); [et al.]. (1999). La Educación en el siglo XXI. Los retos del futuro inmediato. Serie Pedagogía teoría y práctica. Barcelona, España: Editorial Graó, Biblioteca de Aula.

Mertens, Leonard. (1996). Competencia laboral: sistemas, surgimiento y modelos. Montevideo: Cinterfor.

(1998). La Gestión por competencia Laboral en la Empresa y la Formación Profesional. Madrid, España: IBERFOP.

Mora V., Ana Isabel; Herrera P., María Eugenia. (1998). Lineamientos metodológicos para elaborar el perfil académico profesional. San José, C.R.: Universidad de Costa Rica, IIMEC. 
Morales, Damaris. (s.f.). Desarrollo del Talento Humano basado en Competencias Laborales: el Nuevo enfoque de la formación profesional. Ponencia presentada en el Primer Congreso Nacional de Competencias. San José, C.R.: Instituto Nacional de Aprendizaje.

Nirenberg, Olga; Brawerman, Josette; Ruiz, Violeta. (2000). Evaluar para la transformación. México: Paidós.

Pallán, Carlos; Van der Donckt, Pierre (editores). (1995). Evaluación de la calidad y gestión del cambio. Memoria del seminario interamericano "Evaluación de la calidad y gestión de cambio. México: ANUIES.

(1995). Evaluación de la educación superior como estrategia para el cambio. El proceso de autoevaluación. Evaluación de la calidad y gestión del cambio. México: ANUIES.

Pereira, Rocío. (1991). Planeamiento y Evaluación Educativa. San José, Costa Rica: MEP-C.I.P.E.T.

Pérez, Sergio. (s.f.). La investigación y la práctica pedagógica de avanzada. Caminos Abiertos, Educación 23.

Popovsky, Ricardo. (1993). La calidad de la enseñanza. Resultado del seminario temático organizado por la Universidad de Palermo: 6 de agosto de 1992. Canadá, Organización Universitaria Interamericana, Instituto de Gestión y Liderazgo Universitario: Revista IGLU, No.4, Abril.

Posner, George. (1998). Análisis del curriculum. Colombia: McGraw Hill.

Quintana, Juan. (1999). Plan de estudios fundamentado en competencias. Santa Fe de Bogotá: Universidad Militar Nueva Granada.

Rama, Germán. (1989). Cambio social, educación y crisis en América Latina. Análisis, No. 49-50, enero-diciembre.

Román, Marcela. (1999). Hacia una Evaluación Constructivista de Proyectos Sociales. Revista Mad. No.1. Septiembre.

Scott, P. (1990). Introducción a la Investigación y a la Evaluación. Guatemala: Universidad de San Carlos.

Sol, Ricardo. (1995). Reflexiones sobre la gestión del cambio: paradojas, criterios y retos. Evaluación de la calidad y gestión del cambio. Pallán, Carlos y Van der Donckt, Pierre (editores). México: ANUIES.

Soria, Oscar. (1993). El dilema entre saber, poder y querer ¿Una nueva universidad para el siglo XXI?. PLANIUC, Vol.12, No.20.

Stufflebeam, Daniel; Shinkfield, Anthony. (1995). Evaluación sistemática - Guía teórica y práctica. España: Centro de Publicaciones del Ministerio de Educación y Ciencia, Ediciones Paidós Ibérica. 
Tiana, Alejandro. (1996). La evaluación de los sistemas educativos. Revista lberoamericana de Educación, Organización de Estados Iberoamericanos No.10, pp. 37-61.

Toranzos, Lilia. (1996). Evaluación y calidad. Revista Iberoamericana de Educación. Organización de Estados Iberoamericanos No.10, pp. 63-78.

Torrado, María Cristina. (1996). De la evaluación de aptitudes a la evaluación de competencias. Serie Investigación y Evaluación Educativa. La educación superior. Santa Fe de Bogotá: ICFES.

UNESCO. (1990). Libertad creadora y desarrollo humano en una cultura de paz. Nuevos roles de la Educación Superior. Perfiles Educativos, No. 49-50.

Universidad de Costa Rica. Consejo Universitario. (1990). Estatuto Orgánico. San José, Costa Rica: Oficina de Publicaciones de la Universidad de Costa Rica.

Universidad de Costa Rica. Vicerrectoría de Docencia. (1995). Políticas y normas curriculares para la actualización de planes de estudios. San José, C.R.: Oficina de Publicaciones de la Universidad de Costa Rica.

Universidad de Costa Rica. Vicerrectoría de Docencia, Centro de Evaluación Académica. (1999). El proceso de autoevaluación para la acreditación: una guía orientadora. San José, C.R.: Oficina de Publicaciones de la Universidad de Costa Rica.

(1991). Guía para la elaboración y presentación de modificaciones a planes de estudios. San José, C.R.: Oficina de Publicaciones de la Universidad de Costa Rica.

Van Der Donckt, Pierre. (1995). Canadá: estado de la educación superior y, evaluación de la calidad académica y acreditación. Seminario: La acreditación universitaria en el contexto internacional: tendencias, problemas y alternativas de solución. Bogotá.

Vigotski, L.S. (1993). Obras Escogidas. Volumen III. Moscú: Editorial Pedagógica Moscú.

Villarini, Ángel. (1996). El currículo orientado hacia el desarrollo integral. Río Piedra, Puerto Rico: Biblioteca del Pensamiento Crítico.

Yus, Rafael. (1998). Temas transversales: Hacia una nueva escuela. Barcelona, España: Editorial Graó, Serie Transversalidad. 